\title{
Eccentric Mitral Regurgitation Jets Among Patients Having Sustained Inferior Wall Myocardial Infarction
}

\author{
Gabriel S. Levi, B.A., Steven F. Bolling, M.D.,* and David S. Bach, M.D.
}

Department of Medicine, Division of Cardiology and *Department of Surgery, Section of Cardiac Surgery, University of Michigan, Ann Arbor, Michigan

A strong association has been recognized between partial or complete mitral leaflet flail and highly eccentric mitral regurgitation jets. In light of anecdotal observation of eccentric mitral regurgitation apparently due to geometric and functional changes accompanying inferior wall myocardial infarction, the present study was performed to systematically study the eccentricity of mitral regurgitation jets complicating nonacute inferior wall myocardial infarction. Forty-eight consecutive patients with evidence of prior isolated inferior wall myocardial infarction and at least moderate mitral regurgitation but without other valvular, annular, chordal, or ventricular pathology potentially contributory to mitral regurgitation were studied. Mitral regurgitation jets were characterized with respect to eccentricity and anterior versus posterior direction. Regurgitant jet and mitral leaflet position were quantified relative to the mitral annulus. Five of 48 patients (10.4\%) had eccentric jets, of which four were directed posterior and one anterior. Although not reaching statistical significance, patients with eccentric jets tended to have somewhat smaller left atrial size $(41.2 \pm 7.8$ vs $47.2 \pm 9.3 \mathrm{~mm}, \mathrm{P}=0.17)$ and left ventricular size $(51.5 \pm 3.4$ vs $55.1 \pm 7.8 \mathrm{~mm}, \mathrm{P}=0.13)$, and higher left ventricular ejection fraction (0.52 \pm 0.11 vs $0.46 \pm 0.09, \mathrm{P}=0.25)$ compared with patients with noneccentric jets. Leaflet position relative to the mitral annulus was significantly different among patients with eccentric compared with noneccentric posterior jets $\left(54 \pm 10^{\circ}\right.$ vs $\left.33 \pm 11^{\circ}, \mathrm{P}=0.02\right)$, implying greater leaflet restriction toward the left ventricular apex. In conclusion, approximately one in 10 patients with isolated inferior wall myocardial infarction and at least moderate mitral regurgitation was found to have marked eccentricity of the regurgitant jet. Leaflet position was more apically displaced among patients with eccentric jets, suggesting greater leaflet restriction in systole. The finding of a highly eccentric posterior mitral regurgitation jet can be due to inferior wall myocardial infarction with posterior leaflet restriction as well as partial or complete anterior mitral leaflet flail.(ECHOCARDIOGRAPHY, Volume 18, February 2001)

mitral regurgitation, coronary artery disease, echocardiography/Doppler

Echocardiography with color flow Doppler is commonly used to evaluate the etiology as well as the presence and severity of mitral regurgitation, with impact on decisions regarding the necessity, timing, and nature of surgical intervention. In addition to valvular anatomy visualized on echocardiographic imaging, the appearance of a mitral regurgitation jet on color flow Doppler imaging has been found to impart additional information with respect to underlying valvular and nonvalvular pathology..$^{-3}$ An especially strong correlation has been pre-

Address for correspondence and reprint requests: David S. Bach, M.D., L3119 Women's-0273, 1500 E. Medical Center Drive, Ann Arbor, MI 48109-0273. Fax: 734-763-7390; E-mail:dbach@umich.edu. viously observed and well described between highly eccentric jets and underlying myxomatous leaflet degeneration with partial or complete mitral leaflet flail. This association is sufficiently strong that a finding of highly eccentric mitral regurgitation jet can be taken as strong suggestive evidence of partial mitral leaflet flail.

A mitral regurgitation jet that occurs in the setting of leaflet flail is eccentric because of the shape of the regurgitant orifice, such that in systole the flail segment is located "behind" or toward the left atrium relative to the opposing segment of the nonflail leaflet. ${ }^{3}$ This leads to a well recognized pattern in which an eccentric mitral regurgitation jet is directed opposite the affected leaflet: posterior leaflet flail results in 
an eccentric anterior directed jet, and anterior leaflet flail results in an eccentric posterior directed jet. Because coaptation of the mitral valve leaflets is affected by annular, chordal, papillary muscle, and left ventricular anatomy and geometry as well as mitral leaflet anatomy, subvalvular and specifically left ventricular pathology can cause incomplete mitral leaflet coaptation and mitral regurgitation.4-6 To what degree pathologic conditions other than leaflet flail cause eccentric regurgitant jets has not to our knowledge been previously investigated. Although typically associated with central or posterior directed jets, we have observed anecdotal cases in which geometric and functional changes accompanying inferior wall myocardial infarction are associated with highly eccentric mitral regurgitation jets. The purpose of the present study was to systematically study the eccentricity of mitral regurgitation jets complicating nonacute inferior wall myocardial infarction in an attempt to define the prevalence of highly eccentric jets associated with this condition.

\section{Methods}

\section{Study Population}

The University of Michigan Echocardiography Laboratory database was retrospectively searched to identify patients having undergone transthoracic echocardiography and who had both significant mitral regurgitation and evidence of prior isolated inferior wall myocardial infarction. Patients were excluded from analysis if there was evidence of other pathology that could contribute to the presence or severity of mitral regurgitation, including primary leaflet pathology, significant annular calcification, or cardiomyopathy. Specifically, patients were included if there was at least moderate mitral regurgitation; the mitral valve leaflets and annulus were anatomically normal in appearance; left ventricular ejection fraction was $\geq 35 \%$; and there was akinesis of the inferior and/or inferoposterior wall with abnormal wall motion limited to the inferior wall, inferoposterior wall, and proximal inferoseptum. Fortyeight consecutive patients meeting these criteria were evaluated.

\section{Echocardiographic Analysis}

All echocardiograms were performed for clinical indications using commercially available equipment. Two-dimensional and color flow
Doppler imaging were performed from parasternal, apical and, subcostal windows using multiple imaging planes. Images were recorded on standard VHS videotape. Clinical analysis was performed at the time of study by experienced cardiologists with special expertise in echocardiography. Mitral regurgitation was graded as absent, trivial (physiologic), mild, moderate, or severe based on the size and characteristics of the jet on color flow Doppler imaging, ${ }^{7,8}$ in keeping with standard clinical practice. It is the practice of the laboratory to take into consideration jet eccentricity in the clinical estimation of mitral regurgitation severity. Typically, the severity of mitral regurgitation based on jet size relative to left atrial size was increased one grade for eccentric jets. ${ }^{8}$ Standard left atrial and left ventricular dimensions were made from two-dimensional images in the parasternal long-axis view. Left ventricular ejection fraction was calculated using a modified Simpson's rule from apical images. ${ }^{9}$ Left ventricular wall motion analysis used the standard 16-segment model recommended by the American Society of Echocardiography. ${ }^{9}$

Original videotapes were subsequently retrieved for qualitative and quantitative analysis of mitral regurgitation jet eccentricity. All echocardiograms were reviewed and analyzed by a single observer. A mitral regurgitation jet was designated "eccentric" if it originated in the same plane or close to the same plane as the body of a mitral leaflet during systole. All other mitral regurgitation jets were designated as "noneccentric."

\section{Quantitative Analysis}

Schematic demonstration of the quantitative assessment of leaflet and jet angles is shown in Figure 1. Mitral regurgitation jet direction was defined as "anterior" or "posterior" based on its position relative to the anterior and posterior mitral leaflets and the long axis of the left ventricle. Quantitative analysis of mitral regurgitation jet eccentricity was accomplished by comparison of the position of the mitral regurgitation jet with the position of the closest mitral leaflet at the time of jet visualization in systole, using internal cardiac references for position and direction. A single imaging plane was selected for each patient that best displayed the mitral regurgitation jet at its origin, and representative images were digitized from videotape using an offline digital work station (Tomtec, Inc., Boulder, CO, USA). Working 

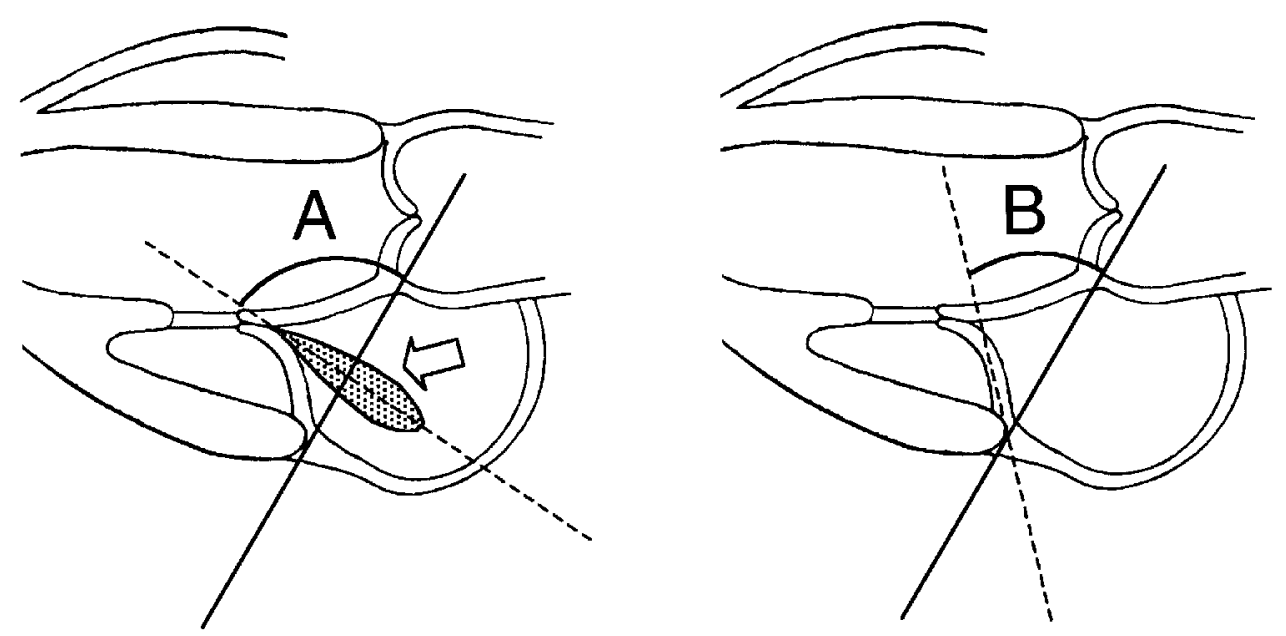

Figure 1. Schematic drawing demonstrating technique for determining mitral regurgitation jet (open arrow, left panel) and mitral leaflet (right panel) position relative to mitral annulus. A line (solid line) connects the anterior and posterior annulus. Mitral regurgitation and mitral leaflet positions are marked (dashed lines). Angles to the nearest $5^{\circ}$ are determined between annular reference line and position of regurgitant jet (angle $A$ ) and mitral leaflet (angle B).

from the digitized image, a reference line was inscribed between the annular insertion of the anterior and posterior mitral leaflets. ${ }^{6} \mathrm{~A}$ second line was defined based on the location and direction of the mitral regurgitation jet at its origin, and the angle between the lines defining the mitral annulus and the origin of the mitral regurgitation jet was determined to the nearest $5^{\circ}$ (Fig. 1, left panel). Finally, a line defining the position of the mitral leaflet body closest to the mitral regurgitation jet was marked at the same time in systole, and the angle between the line defining the leaflet and the reference line defining the mitral annulus was similarly determined to the nearest $5^{\circ}$ (Fig. 1 , right panel). The numerical difference between these two angles was used as an index of jet eccentricity:

Jet eccentricity $=$ Angle 2(annulus-to-jet)

- Angle 1(annulus-to-leaflet).

Using this system, the numerical difference between the two angles is small for eccentric jets and larger for noneccentric jets. A leaflet position parallel with the anterior-posterior mitral annular plane is expressed as a leaflet angle of $0^{\circ}$, whereas systolic leaflet restriction is reflected by a larger leaflet angle relative to the annular reference line. Examples of a noneccentric jet and of posterior leaflet restriction with an eccentric anterior jet are shown schematically in Figure 2.

\section{Intraobserver Variability}

A subset of 12 echocardiograms was randomly selected for assessment of intraobserver variability of quantitative measurements. Measurement of angles between annulus and mitral regurgitation jet and annulus and mitral leaflet was repeated by the same observer without access to prior measurements. Intraobserver variability was defined as the absolute and percent difference between measurements for each patient.

\section{Statistical Analysis}

All data are represented as mean \pm 1 standard deviation. Comparisons between the direction (anterior vs posterior) of mitral regurgitation jets, mitral regurgitation severity, and the prevalence of normal ejection fraction between "eccentric" and "noneccentric" groups were made using Fisher exact tests. Comparison of measured leaflet and jet angles, left atrial and left ventricular dimensions, and left ventricular ejection fraction between "eccentric" and "noneccentric" groups were made using unpaired Student's $t$-tests. Differences were considered significant at a value of $\mathrm{P}<0.05$.

\section{Results}

\section{Intraobserver Variability}

Mitral regurgitation jet and mitral valve leaflet angles were both measured twice among 

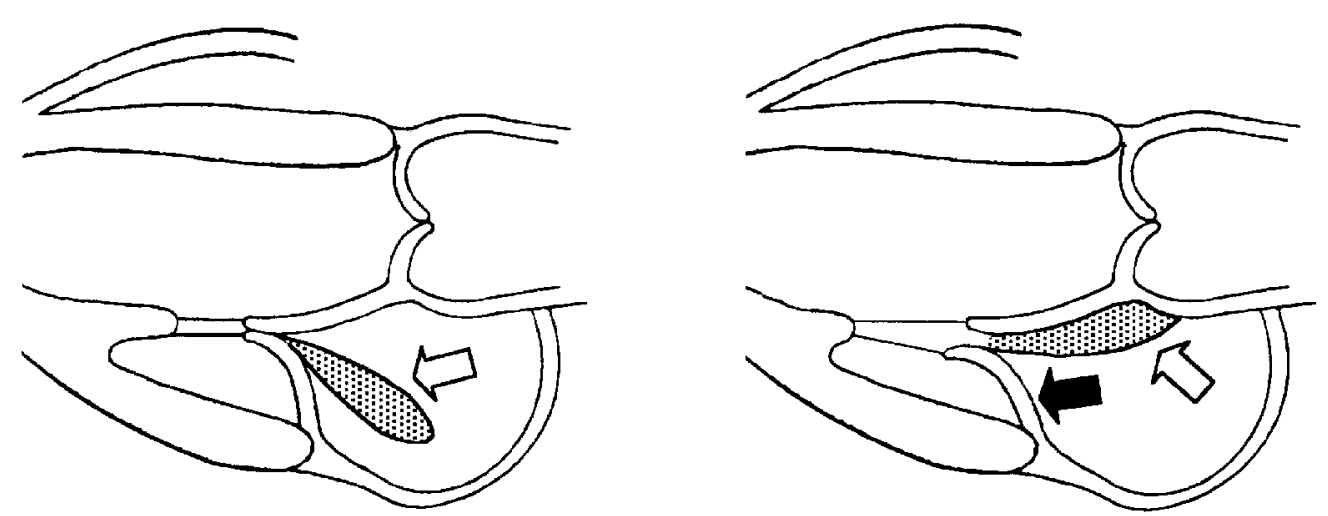

Figure 2. Schematic examples of noneccentric (left panel) and eccentric (right panel) regurgitant jets (open arrows). Right panel shows restriction of posterior mitral leaflet (solid arrow), with eccentric anterior directed mitral regurgitation.

12 patients. There was concordance between measurements (to the nearest $5^{\circ}$ ) for 11 of 24 (46\%) measurements, and the difference between measurements was $\leq 10^{\circ}$ for 21 of 24 $(88 \%)$. The average variability between measurements was $4.8 \pm 6.2^{\circ}(8.9 \pm 10.8 \%)$. There was complete concordance in the assessment of mitral regurgitation jet direction.

\section{Jet Eccentricity}

Of 48 patients studied who met criteria for isolated inferior wall myocardial infarction and at least moderate mitral regurgitation, 5 (10.4\%) met visual criteria for mitral regurgitation jet eccentricity. The severity of mitral regurgitation did not differ significantly between groups; all patients $(100 \%)$ with eccentric jets and 38 of $43(88.4 \%)$ patients with noneccentric jets had moderate mitral regurgitation $(\mathrm{P}=1.00)$; the remaining five patients with noneccentric jets had severe regurgitation.

Of five patients with eccentric mitral regurgitation jets, four jets (80\%) were directed posterior whereas only one (20\%) was directed anterior. Quantitative determination of jet eccentricity was concordant with visual assessment and is shown in Table I. Although the prevalence of jets that were directed relatively posterior to midline did not differ between "eccentric" and "noneccentric" groups, jet angles relative to the annular reference line and quantitative measures of jet eccentricity were significantly different between groups.

\section{Left Ventricular Size and Function}

Measures of left atrial size and left ventricular size and systolic function are also shown in Table I. There were no statistically significant differences between "eccentric" and "non-

TABLE I

Comparison of Jet Angle, Leaflet Angle, Cardiac Dimensions, and Left Ventricular Ejection Fraction for all Patients, Divided by "Eccentric" and "Noneccentric" Jets

\begin{tabular}{lccc}
\hline & "Eccentric" & "Noneccentric" & $\mathrm{P}$ \\
\hline $\mathrm{n}$ & 5 & 43 & \\
Posterior jet direction & $4(80 \%)$ & $36(84 \%)$ & 1.00 \\
Leaflet angle & $49 \pm 14^{\circ}$ & $33 \pm 11^{\circ}$ & 0.06 \\
Jet angle & $51 \pm 12^{\circ}$ & $77 \pm 11^{\circ}$ & 0.01 \\
Difference (Jet angle-leaflet angle) & $4 \pm 2^{\circ}$ & $44 \pm 12^{\circ}$ & $<0.001$ \\
LA size (mm) & $41.2 \pm 7.8$ & $47.2 \pm 9.3$ & 0.17 \\
LVIDD (mm) & $51.5 \pm 3.4$ & $55.1 \pm 7.8$ & 0.13 \\
LVIDS (mm) & $40.0 \pm 7.1$ & $44.6 \pm 8.3$ & 0.30 \\
LV ejection fraction & $0.52 \pm .11$ & $0.46 \pm 0.09$ & 0.25 \\
\hline
\end{tabular}


TABLE II

Comparison of Jet Angle and Leaflet Angle for Patients with Posterior Directed Mitral Regurgitation Jets, Divided by "Eccentric" and "Noneccentric" Jets

\begin{tabular}{lccc}
\hline & "Eccentric" & "Noneccentric" & $\mathrm{P}$ \\
\hline $\mathrm{n}$ & 4 & 36 & \\
Posterior leaflet angle & $54 \pm 10^{\circ}$ & $33 \pm 11^{\circ}$ & 0.02 \\
Jet angle & $55 \pm 9^{\circ}$ & $78 \pm 11^{\circ}$ & 0.01 \\
Difference (Jet angle—leaflet angle) & $4 \pm 2^{\circ}$ & $45 \pm 12^{\circ}$ & $<0.001$ \\
\hline
\end{tabular}

eccentric" groups, although there were trends that did not reach statistical significance toward somewhat smaller left atrial size, smaller systolic and diastolic left ventricular dimensions, and somewhat higher left ventricular ejection fraction among patients with eccentric mitral regurgitation jets compared with those with noneccentric jets. Similarly, the prevalence of left ventricular ejection fraction within normal limits tended to be higher among those with eccentric compared with noneccentric jets (3 of 5 [60\%] vs 12 of 43 [28\%], P $=0.31$ ), although the difference did not reach statistical significance.

\section{Leaflet Position}

Only one patient with eccentric mitral regurgitation had an anterior directed jet. The position of this patient's anterior mitral leaflet was $30^{\circ}$ from the annular reference line, similar to the $34 \pm 9^{\circ}$ anterior leaflet angle observed among the seven patients with noneccentric anterior jets. Although the single patient precludes performance of statistical analysis, the jet angle for the patient with eccentric anterior mitral regurgitation appeared substantially different than that observed among the seven patients with anterior noneccentric jets $\left(35^{\circ} \mathrm{vs}\right.$ $71 \pm 8^{\circ}$ ). Similarly, the difference between jet and leaflet angles appeared to be substantially smaller in this patient than among the other seven $\left(5^{\circ}\right.$ vs $\left.37 \pm 7^{\circ}\right)$.

Leaflet and jet positions for patients with a posterior directed regurgitant jet are shown in Table II. In distinction to the patients with anterior jets, posterior leaflet position was significantly different between groups based on jet eccentricity; patients with eccentric posterior directed jets had a greater angle between the posterior mitral leaflet and the annular reference line. The larger angle relative to the mitral annulus corresponds to a leaflet position closer to parallel with the long axis of the left ventricle in patients with eccentric jets, suggesting greater systolic leaflet restriction than among those patients with noneccentric jets.

\section{Discussion}

\section{Jet Eccentricity Following Inferior Infarction}

A strong association exists between flail or partially flail mitral leaflet and eccentric regurgitant jets. This association is sufficiently strong that the presence of an eccentric mitral regurgitation jet can be taken as evidence of underlying leaflet flail. ${ }^{3}$ In the present study, 5 of 48 patients, or approximately 1 in 10 with isolated inferior wall myocardial infarction and at least moderate mitral regurgitation studied in the nonacute setting had evidence of a highly eccentric mitral regurgitation jet. Among these patients, the mechanism of mitral regurgitation is presumably due to restricted systolic mobility of a mitral leaflet caused by geometric changes in left ventricular size and shape, without primary leaflet pathology. 4-6 Inasmuch as an etiology other than leaflet flail can result in a highly eccentric mitral regurgitation jet, the finding of jet eccentricity on color flow Doppler imaging should not be taken as synonymous with an underlying pathology of flail leaflet.

Although the present study was performed in a nonacute setting, it is possible that a similar finding could be observed among patients suffering acute inferior wall myocardial infarction. In this setting, unfavorable left ventricular remodeling with early infarct expansion could result in eccentric mitral regurgitation. Because of distinct clinical implications, eccentric mitral regurgitation caused by leaflet restriction should be differentiated from acute papillary muscle rupture with leaflet flail.

\section{Geometric and Functional Features}

Incomplete mitral leaflet coaptation due to changes in left ventricular size, shape, and 
function have been previously shown to cause mitral regurgitation among patients with myocardial ischemia despite normal leaflet and annular anatomy.5,6 In the present study, patients with ischemic mitral regurgitation that assumed an eccentric jet direction did not have significantly different measures of left atrial size or left ventricular size or systolic function compared with those with noneccentric mitral regurgitation jets. However, there were trends that did not reach statistical significance toward slightly smaller left atrial and left ventricular size and slightly higher left ventricular ejection fraction among patients with eccentric compared with noneccentric jets. The severity of mitral regurgitation did not differ significantly between groups, with the preponderance of patients having moderate mitral regurgitation, suggesting that ventricular size, geometry, and/or function could play a role in jet eccentricity. Specifically, although not reaching statistical significance, trends observed in the present study suggest that patients having suffered a smaller inferior wall myocardial infarction, with higher residual left ventricular ejection fraction, may be more prone to the specific geometric changes that result in an eccentric mitral regurgitation jet.

Of interest, posterior mitral leaflet position relative to the mitral annulus was significantly different among patients with eccentric compared with noneccentric mitral regurgitation. The larger measured angle relative to the mitral annulus among patients with eccentric $\left(54 \pm 10^{\circ}\right)$ compared with noneccentric jets $\left(33 \pm 11^{\circ}\right)$ corresponds to a leaflet position further displaced from the plane of the mitral annulus, presumably caused by greater leaflet restriction toward the left ventricular apex. Kono et al. ${ }^{6}$ previously described the role that mitral leaflet restriction plays in incomplete mitral leaflet coaptation and ischemic mitral regurgitation. The present study suggests that varying degrees of leaflet restriction, accompanied by other variables potentially including left ventricular and annular size and left ventricular systolic function, may be responsible for mitral regurgitation jets of varying eccentricity.

\section{Study Limitations}

For purposes of determining relative positions of mitral leaflet and mitral regurgitation jet, a line was drawn between the anterior and posterior mitral annulus. Although this system ignores the saddle shape of the mitral annulus, the line served only as an internal reference; the same reference in the same view was used for determination of both mitral leaflet and regurgitant jet positions.

Mitral regurgitation volume was not quantified beyond the clinical assessment of mild, moderate, or severe. Although the area of a jet on color flow Doppler imaging is known to understate the severity of eccentric compared with central regurgitant jets, ${ }^{8}$ such factors were taken into consideration in the clinical assessment of mitral regurgitation severity. Further, the primary study finding that eccentric mitral regurgitation occurs in the setting of inferior wall myocardial infarction without leaflet flail is not impacted by the quantification of regurgitation.

The present study was limited to the assessment of mitral leaflet position relative to the mitral annulus. Other geometric and functional features of the mitral apparatus and left ventricle were not quantified and are outside the scope of this study. Finally, pathologic correlation was not available in the present study but could be the subject of future investigation.

\section{Conclusions}

There is a strong and well-established association between flail mitral leaflet and eccentric mitral regurgitation. However, a markedly eccentric mitral regurgitation jet was found in approximately one in 10 patients with isolated inferior wall myocardial infarction and at least moderate mitral regurgitation. A majority of such eccentric jets were directed posterior. Among these patients, the mechanism of mitral regurgitation appears to be related to incomplete mitral leaflet coaptation due to posterior leaflet restriction. The finding of a highly eccentric posterior mitral regurgitation jet can be due to inferior wall myocardial infarction with posterior leaflet restriction as well as to partial or complete anterior mitral leaflet flail.

\section{References}

1. Grayburn PA, Berk MR, Spain MG, et al: Relation of echocardiographic morphology of the mitral apparatus to mitral regurgitation in mitral valve prolapse: Assessment by Doppler color flow imaging. Am Heart $J$ 1990;119:1095-1102.

2. Pearson AC, St. Vrain J, Mrosek D, et al: Color Doppler echocardiographic evaluation of patients with a flail mitral leaflet. J Am Coll Cardiol 1990; 16:232-239. 


\section{ECCENTRIC MITRAL REGURGITATION}

3. Stewart WJ, Currie PJ, Salcedo EE, et al: Evaluation of mitral leaflet motion by echocardiography and jet direction by color Doppler flow mapping to determine the mechanism of mitral regurgitation. J Am Coll Cardiol 1992;20:1353-1361.

4. Kaul S, Pearlman JD, Touchstone DA, et al: Prevalence and mechanism of mitral regurgitation in the absence of intrinsic abnormalities of the mitral leaflets. Am Heart J 1989;118:963-971.

5. Kaul S, Spotnitz WD, Glasheen WP, et al: Mechanism of ischemic mitral regurgitation: An experimental evaluation. Circulation 1991;84:2167-2180.

6. Kono T, Sabbah HN, Rosman H, et al: Mechanism of functional mitral regurgitation during acute myo- cardial ischemia. J Am Coll Cardiol 1992;19:11011105.

7. Spain MG, Smith MD, Grayburn PA, et al: Quantitative assessment of mitral regurgitation by Doppler color flow imaging: Angiographic and hemodynamic correlations. J Am Coll Cardiol 1989;13:585-590.

8. Cape EG, Yoganathan AP, Weyman AE, et al: Adjacent solid boundaries alter the size of regurgitant jets on Doppler color flow maps. J Am Coll Cardiol 1991;17: 1094-1102.

9. Schiller NB, Shah PM, Crawford M, et al: Recommendations for quantitation of the left ventricle by twodimensional echocardiography. J Am Soc Echocardiogr 1989;2:358-367. 